OPEN ACCESS

Edited by: Umberto Malapelle,

University of Naples Federico II, Italy

Reviewed by: Jelena Stojsic,

University of Belgrade, Serbia

Pasquale Pisapia,

University of Naples Federico II, Italy

*Correspondence:

Foteinos-Ioannis D. Dimitrakopoulos fodimitrakopoulos@upatras.gr

Haralabos P. Kalofonos

kalofonos@upatras.gr

${ }^{+}$These authors have contributed equally to this work and share first authorship

Specialty section: This article was submitted to Thoracic Oncology, a section of the journal Frontiers in Oncology

Received: 07 June 2021 Accepted: 10 August 2021 Published: 17 September 2021

Citation: Dimitrakopoulos F-ID, Antonacopoulou AG, Kottorou AE, Kalofonou M, Panagopoulos N, Dougenis D, Makatsoris T, Tzelepi V, Koutras A and Kalofonos HP (2021) Genetic Variations of CD40 and LTRR Genes Are Associated With Increased Susceptibility and Clinical Outcome of Non-Small-Cell Carcinoma Patients.

Front. Oncol. 11:721577. doi: 10.3389/fonc.2021.721577

\section{Genetic Variations of CD40 and $L T \beta R$ Genes Are Associated With Increased Susceptibility and Clinical Outcome of Non-Small-Cell Carcinoma Patients}

\author{
Foteinos-loannis D. Dimitrakopoulos ${ }^{1,2 *}$, Anna G. Antonacopoulou ${ }^{2 \dagger}$, \\ Anastasia E. Kottorou ${ }^{2}$, Melpomeni Kalofonou ${ }^{3}$, Nikolaos Panagopoulos ${ }^{4}$, \\ Dimitrios Dougenis ${ }^{4}$, Thomas Makatsoris ${ }^{1}$, Vasiliki Tzelepi ${ }^{5}$, Angelos Koutras ${ }^{1}$ \\ and Haralabos P. Kalofonos ${ }^{1 *}$

\begin{abstract}
${ }^{1}$ Division of Oncology, Department of Medicine, University of Patras, Patras, Greece, ${ }^{2}$ Molecular Oncology Laboratory, Division of Oncology, Department of Medicine, University of Patras, Patras, Greece, ${ }^{3}$ Centre for Bio-Inspired Technology, Institute of Biomedical Engineering, Imperial College London, London, United Kingdom, ${ }^{4}$ Department of Cardiothoracic Surgery, University of Patras, Patras, Greece, ${ }^{5}$ Department of Pathology, University of Patras, Patras, Greece
\end{abstract}

Background: Immune system-related receptors CD40 (tumor necrosis factor receptor superfamily member 5), BAFFR (tumor necrosis factor receptor superfamily member 13C), and LT $\beta R$ (tumor necrosis factor receptor superfamily member 3) play a pivotal role in nonsmall-cell lung cancer (NSCLC). To further evaluate their role in NSCLC, CD40 rs1883832 $(T>C)$, BAFFR rs7290134 (A>G), and $L T \beta R$ rs10849448 (A>G) single-nucleotide polymorphisms (SNPS) were investigated regarding their impact in risk and clinical outcome of NSCLC patients.

Methods: The three selected SNPs were evaluated in 229 NSCLC patients and 299 healthy controls, while CD40, BAFFR, and LT $\beta R$ protein expression was assessed by immunohistochemistry in 96 tumor specimens from NSCLC patients.

Results: In total, CD40 rs1883832 was associated with NSCLC risk, with the T allele, after adjusting for cofactors, being related to increased risk ( $p=0.007$; OR 1.701). Moreover, the CT genotype was associated with increased risk ( $p=0.024 ;$ OR 1.606) and poorer 5-year overall survival (OS) after adjusting for cofactors ( $p=0.001, H R 1.829)$, while CC was associated with higher CD40 expression in tumorous cells $(p=0.040)$ and in stromal cells $(p=0.036)$. In addition, AA homozygotes for the LTRR rs10849448 had increased risk for NSCLC in multivariate analysis ( $p=0.008$; OR, 2.106) and higher LTRR membranous expression $(p=0.035)$. Although BAFFR rs7290134 was associated with BAFFR membranous expression ( $p=0.039$ ), BAFFR rs7290134 was not associated with neither the disease risk nor the prognosis of NSCLC patients. 
Conclusions: In conclusion, CD40 rs1883832 and $L T \beta R$ rs10849448 seem to be associated with increased risk for NSCLC, while CD40 rs1883832 is also associated with OS of patients with NSCLC.

Keywords: SNP, NSCLC, rs1883832, rs10849448, rs7290134, prognosis, risk

\section{INTRODUCTION}

The last decade, NF- $\mathrm{KB}$ (nuclear factor kappa-light-chainenhancer of activated B cells) has attracted interest regarding its role in NSCLC (non-small-cell lung cancer) (1). It has been documented that the main effectors of the classical, NF-KB1 and RelA (transcription factor P65), as well as of the alternative pathway, NF- $\mathrm{KB} 2$ and RelB (transcription factor RelB), are overexpressed and have prognostic value in NSCLC (1-5). On the contrary, published data on the pathobiology and the clinical significance in NSCLC of the surface receptors CD40 (tumor necrosis factor receptor superfamily member 5), BAFFR (tumor necrosis factor receptor superfamily member $13 \mathrm{C}$ ), and LT $\beta R$ (tumor necrosis factor receptor superfamily member 3), which mainly leads to signal transduction through the NF- $\kappa B$ alternative pathway, are limited. Recently, our group has reported that these receptors are expressed in NSCLC revealing significant clinical associations (6). According to our studies as well as to other studies, CD40 expression in lung cancer patients has been associated with metastatic progression (7) as well as with prognosis $(6,8)$. Similarly, BAFFR expression in NSCLC has also been reported to be deregulated (6), while its expression in CAFs (cancer-associated fibroblasts) has interestingly been associated with overall survival (OS) and response to platinum-based chemotherapy in NSCLC (9).

More limited are also the published data on genetic variations of $C D 40, B A F F R$, and $L T \beta R$ and especially of the three singlenucleotide polymorphisms (SNPs) CD40 rs1883832 (T>C), BAFFR rs7290134 (A>G), and LT $\beta R$ rs10849448 (A>G), which were evaluated in the current study, as well as their potent clinical value in NSCLC. In particular, CD40 SNP rs1883832 has been associated with many nonmalignant clinical entities, such as atherosclerosis (10), acute coronary syndrome $(11,12)$, ischemic stroke (13), chronic obstructive pulmonary disease (14), chronic HBV infection (15), and later onset of Graves' disease (16). It has also been related to cervical carcinoma in a subset of a Malaysian population (17) and to sporadic breast cancer risk in Chinese Han women (18). Interestingly, in a small study in the Chinese population, it has also been correlated with the susceptibility to lung cancer (19). Regarding the BAFFR rs7290134, no association with the risk for CLL (chronic lymphocytic leukemia) was found $(20,21)$, while no study has been published regarding its role in epithelial tumors. In addition, the published data regarding the clinical value of $L T \beta R$ rs $10849448(A>G)$ refer to its association with individuals' risk of undergoing tonsillectomy (22) and juvenile idiopathic arthritis (23), while no data exist regarding its significance in cancer.

In the present study, we present our findings on the clinical significance of CD40 rs1883832 (T>C), BAFFR rs7290134
$(\mathrm{A}>\mathrm{G})$, and $L T \beta R$ rs $10849448(\mathrm{~A}>\mathrm{G})$ SNPs in NSCLC patients in regard to the risk over NSCLC initiation and OS.

\section{MATERIALS AND METHODS}

\section{Study Design, Population, Tissue Specimens, and Data Collection}

The current study was performed following Helsinki Declaration on ethical principles for medical research (2013) (24). In this study, we investigated the selected SNPs of CD40, BAFFR, and $L T \beta R$ in relation to clinicopathological data, protein expression of the same molecules, and the clinical outcome of NSCLC patients. Initially, the selected SNPs were studied in a retrospectively collected group which was followed by a second prospectively collected patient cohort. Although our preliminary results from the retrospective group were validated in the prospective group, statistical analysis presented here was performed in the pool of the cases to achieve more robust statistical results.

Data (clinicopathological, disease outcome, and vital status) used in the analysis were obtained from pathology reports, from medical records, or through direct communication with the patients. Survival outcome was evaluated after the 60-month follow-up period. Clinicopathological characteristics of our cohort and relevant information are included in Table 1.

Histopathologically or cytologically confirmed NSCLC (squamous cell carcinoma or adenocarcinoma or large-cell carcinoma) patients were exclusively enrolled in the study. Totally, 229 previously untreated patients with confirmed NSCLC of all ages were enrolled, with 109 of them being retrospectively collected (Group R). Tissue specimens from the patients were collected from the archives of the Department of Pathology of the University Hospital of Patras, Greece: tumoradjacent, nonmalignant, and macroscopically normal tissue specimens. Additionally, peripheral blood specimens from 120 newly diagnosed NSCLC patients, which were managed from 2008 to 2010, were prospectively collected (Group P). All cases were diagnosed and medically managed at the Division of Medical Oncology and the Department of Cardiothoracic Surgery at the University Hospital of Patras between 2004 and 2015. All patients of the study were treated based on the standard-of-care treatment options according to disease characteristics, the comorbidities, and the performance status following the current treatment guidelines. Uncertain histology, non-Greek Caucasian ethnic origin, and past medical history with cancer were the exclusion criteria for this study.

A healthy control group was also used, including 299 peripheral blood specimens from healthy control donors 
TABLE 1 | Clinicopathological characteristics of patients of this study.

\begin{tabular}{|c|c|c|c|}
\hline Clinicopathological characteristics & $\begin{array}{c}\text { Retrospectively collected cases (Group R) } \\
\text { Cases } \\
n(\%)\end{array}$ & $\begin{array}{c}\text { Prospectively collected cases (Group P) } \\
\text { Cases } \\
n(\%)\end{array}$ & $\begin{array}{c}\text { Total cases (Group PR) } \\
\text { Cases } \\
n(\%)\end{array}$ \\
\hline Total & $109(100)$ & $120(100)$ & $229(100)$ \\
\hline Age (years) Median (range) & $67(46-84)$ & $66.5(41-84)$ & $67(41-84)$ \\
\hline \multicolumn{4}{|l|}{ Gender } \\
\hline Total & 109 (100) & $120(100)$ & $229(100)$ \\
\hline Male & $100(91.7)$ & $108(90.0)$ & $208(90.8)$ \\
\hline Female & $9(9.3)$ & $12(10.0)$ & 21 (9.2) \\
\hline \multicolumn{4}{|l|}{ Smoking (pack-years) } \\
\hline Total & $109(100)$ & $120(100)$ & $229(100)$ \\
\hline Cases (\%) & $47(43.1)$ & $43(35.8)$ & $90(39.3)$ \\
\hline Mean (range) & $89.4(20-165)$ & 84.65 (10-200) & $87.1(10-200)$ \\
\hline NA & $62(56.9)$ & 77 (64.2) & $139(60.7)$ \\
\hline \multicolumn{4}{|l|}{ Primary location } \\
\hline Total & $109(100)$ & $120(100)$ & $229(100)$ \\
\hline Left lung & $49(45.0)$ & $51(42.5)$ & $100(43.7)$ \\
\hline Right lung & $60(55.0)$ & $61(50.8)$ & $121(52.8)$ \\
\hline NA & - & $8(6.7)$ & $8(3.5)$ \\
\hline \multicolumn{4}{|l|}{ Histology } \\
\hline Total & $109(100)$ & $120(100)$ & $229(100)$ \\
\hline Squamous & 65 (59.6) & $46(38.3)$ & $111(48.5)$ \\
\hline Adenocarcinoma & $36(33.0)$ & $62(51.7)$ & $98(42.8)$ \\
\hline Large carcinoma & $7(6.4)$ & $5(4.2)$ & $12(5.2)$ \\
\hline NA & $1(0.9)$ & $7(5.8)$ & $8(0.04)$ \\
\hline \multicolumn{4}{|l|}{ Stage } \\
\hline Total & $109(100)$ & $120(100)$ & $229(100)$ \\
\hline I & 37 (33.9) & $21(17.5)$ & $58(25.3)$ \\
\hline$\|$ & $32(29.4)$ & $19(15.8)$ & $51(22.3)$ \\
\hline III & 37 (33.9) & $32(26.7)$ & $69(30.1)$ \\
\hline IV & $2(1.8)$ & $37(30.8)$ & $39(17.0)$ \\
\hline NA & $1(0.9)$ & $11(9.2)$ & $12(5.2)$ \\
\hline \multicolumn{4}{|l|}{ Grade } \\
\hline Total & $109(100)$ & $120(100)$ & $229(100)$ \\
\hline I & $4(3.7)$ & $2(1.7)$ & $6(2.6)$ \\
\hline$\|$ & 45 (41.3) & 40 (33.3) & $85(37.1)$ \\
\hline III & $52(47.7)$ & $38(31.7)$ & 90 (39.3) \\
\hline NA & $8(7.3)$ & 40 (33.3) & $48(21)$ \\
\hline \multicolumn{4}{|l|}{ Maximum diameter $(\mathrm{cm})$} \\
\hline Total & $109(100)$ & $120(100)$ & $229(100)$ \\
\hline Cases (\%) & $106(97.2)$ & $81(67.5)$ & $187(81.7)$ \\
\hline Mean (range) & $5.27(0.70-21.0)$ & $4.33(1.0-10.0)$ & $4.87(0.70-21.0)$ \\
\hline NA & $3(2.8)$ & $39(32.5)$ & $42(18.3)$ \\
\hline \multicolumn{4}{|l|}{ Lymph node infiltration } \\
\hline Total & $109(100)$ & $120(100)$ & $229(100)$ \\
\hline No & $56(51.4)$ & $35(29.2)$ & $91(39.7)$ \\
\hline Yes & $49(45.0)$ & $31(25.8)$ & $80(34.9)$ \\
\hline NA & $4(3.7)$ & $54(45.0)$ & $58(25.3)$ \\
\hline \multicolumn{4}{|l|}{ Metastasis (adrenals) } \\
\hline Total & $109(100)$ & $120(100)$ & $229(100)$ \\
\hline No & $21(19.3)$ & $15(12.5)$ & $36(15.7)$ \\
\hline Yes & $4(4.7)$ & $12(10)$ & $16(7.0)$ \\
\hline NA & $84(77.1)$ & $93(77.5)$ & $177(77.3)$ \\
\hline \multicolumn{4}{|l|}{ Metastasis (liver) } \\
\hline Total & $109(100)$ & $120(100)$ & $229(100)$ \\
\hline No & $22(20.2)$ & $12(10.0)$ & $34(14.8)$ \\
\hline Yes & $3(2.8)$ & $17(14.2)$ & $20(8.7)$ \\
\hline NA & $84(77.1)$ & $91(75.8)$ & $175(76.4)$ \\
\hline \multicolumn{4}{|l|}{ Metastasis (brain) } \\
\hline Total & $109(100)$ & $120(100)$ & $229(100)$ \\
\hline No & $22(20.2)$ & $12(10.0)$ & $34(14.8)$ \\
\hline Yes & $7(6.4)$ & $27(22.5)$ & $34(14.8)$ \\
\hline NA & $80(73.4)$ & $81(67.5)$ & $161(70.3)$ \\
\hline
\end{tabular}


TABLE 1 | Continued

Clinicopathological characteristics

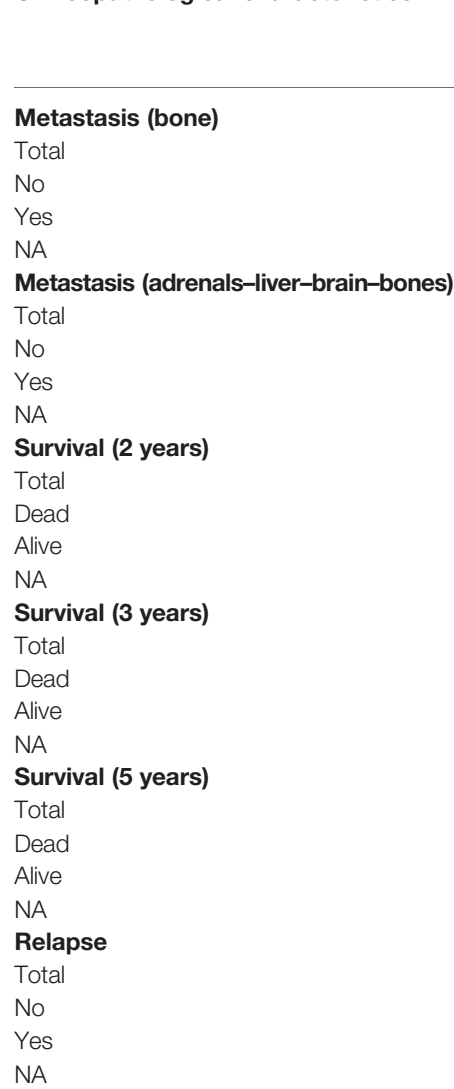

$\begin{array}{ccc}\text { Retrospectively collected cases (Group R) } & \text { Prospectively collected cases (Group P) } & \text { Total cases (Group PR) } \\ \text { Cases } & \text { Cases } & \text { Cases } \\ n(\%) & n(\%) & n(\%)\end{array}$

$n(\%)$

$n(\%)$$$
109(100)
$$$$
67(61.5)
$$$$
38(34.9)
$$$$
4(3.7)
$$$$
109(100)
$$$$
9(8.3)
$$$$
17(15.6)
$$$$
83(76.1)
$$

$$
\begin{gathered}
120(100) \\
14(11.7) \\
26(21.7) \\
80(66.7) \\
120(100) \\
6(5.0) \\
49(40.8) \\
65(54.2) \\
120(100) \\
63(52.5) \\
52(43.3) \\
5(4.2) \\
120(100) \\
77(64.2) \\
37(30.8) \\
6(5.0) \\
120(100) \\
90(75.0) \\
24(20.0) \\
6(5.0) \\
120(100) \\
1(0.8) \\
12(10.0) \\
107(89.2)
\end{gathered}
$$

NA, not available or no further specifically categorized (e.g., NSCLC vs. squamous histology).

(Group H) who were collected in the context of annual medical checkup. Groups of controls and patients were age- and sexmatched (Table 2). In this study, only individuals with Greek Caucasian origin were recruited in order to ensure the similarity of the genetic background. Past medical history related to cancer or family history linked to any disease (including cancer) was set as exclusion criterion.

\section{Selection of SNPs}

The selection of the particular SNPs located within the CD40, $B A F F R$, and $L T \beta R$ genes was performed by reviewing literature (PubMed, Google Scholar) using as keywords the words SNPs, $C D 40, B A F F R, L T \beta R$, cancer, NSCLC, and lung cancer. Five percent was set as the cutoff point for minor allele frequencies,

TABLE 2 | Demographic characteristics of the healthy controls' and NSCLC patients' groups.

\begin{tabular}{lccc}
\hline Groups (N) & $\begin{array}{c}\text { Patients group } \\
(\mathbf{2 2 9 )}\end{array}$ & $\begin{array}{c}\text { Controls group } \\
(\mathbf{2 9 9 )}\end{array}$ & p value \\
\hline Age (years) & $67(41-84)$ & $64(30-95)$ & 0.001 \\
Gender (N males/ & $208 / 21$ & $157 / 142$ & $<0.001$ \\
females) & & &
\end{tabular}

and the coefficient of determination $\mathrm{r}^{2}=0.8$ was captured. Genomic location, minor allele frequencies (MAF), and other specific characteristics of SNPs are presented in Table 3.

\section{DNA Isolation}

The commercial kit "QIAamp Blood Mini Kit" (Qiagen Ltd., Crawley, UK) was used for the extraction of genomic DNA from whole blood samples from 124 cancer patients and 279 healthy donors according to the manufacturer's instructions. Furthermore, DNA was also isolated from 148 tumor adjacent, nonmalignant FFPE, tissue specimens using the "QIAamp DNA FFPE Tissue Kit" (Qiagen Ltd., Crawley, UK). DNA was stored at $-20^{\circ} \mathrm{C}$ until required.

\section{Genotyping}

Genotyping was performed using real-time PCR followed by high-resolution melt curve (HRM) analysis on a StepOnePlus ${ }^{\text {TM }}$ real-time PCR system (Thermo Fisher Scientific, Waltham, MA USA). Reactions were performed using SNP-specific primers (CD40 rs1883832: forward 5'-GCCTGGTCTCACCTCGC-3' and reverse 5'-GCCCCAGAGGACGCAC-3'; BAFFR rs7290134: forward 5'-GCTGAATGCTGTGGTCTGTAGTG-3' and reverse 5'-CATGCACATGCCCTCTTTCTG-3'; LtBR rs10849448 forward 5'-CGGCCAGCTCGCTCCAC-3' and reverse $5^{\prime}$-GCCTCCAGGGCTCCCA-3') and MeltDoctor ${ }^{\mathrm{TM}}$ 
TABLE 3 | Studied SNP information.

\begin{tabular}{|c|c|c|c|c|c|c|c|c|}
\hline \multirow[t]{2}{*}{ Gene } & \multirow{2}{*}{$\begin{array}{l}\text { Gene } \\
\text { location }\end{array}$} & \multirow[t]{2}{*}{ Rs number } & \multirow[t]{2}{*}{ Base change } & \multirow{2}{*}{$\begin{array}{l}\text { Genomic position } \\
\text { (forward strand) }\end{array}$} & \multirow[t]{2}{*}{ Genotyping success rate } & \multicolumn{3}{|c|}{ Minor allele frequency } \\
\hline & & & & & & Control & 1000genomes-CEU & 1000genomes TSI- \\
\hline CD40 & 5 prime UTR & rs1883832 & $\mathrm{T} / \mathrm{C}$ & 20:46118343 & 99.8 & $0.441(T)$ & $0.232(T)$ & $0.299(T)$ \\
\hline BAFFR & 3 prime UTR & rs7290134 & $\mathrm{A} / \mathrm{G}$ & 22:41925247 & 100 & $0.187(G)$ & $0.247(G)$ & $0.215(G)$ \\
\hline LtBR & 5 prime UTR & rs10849448 & $A / G$ & $12: 6384185$ & 100 & $0.313(\mathrm{~A})$ & $0.227(\mathrm{~A})$ & $0.294(A)$ \\
\hline
\end{tabular}

HRM Master Mix (Thermo Fisher Scientific, Waltham, MA USA). HRM genotyping data were analyzed using the HighResolution Melt Software v3.0 (Applied Biosystems, Thermo Fisher Scientific, Waltham, MA USA).

\section{Sequencing}

Further validation of the genotyping method as well as genotyping in some subgroups of participants due to the observed deviation from the Hardy-Weinberg equilibrium (HWE) was achieved through sequencing of several samples, representative of all three genotypes. The sequences of the primers and PCR conditions that were used for sequencing can be provided upon request. Sequencing was performed at Cemia SA (University of Thessaly, Greece). All obtained sequences were in agreement with the genotyping results of our method.

\section{Immunohistochemical Analysis}

CD40, BAFFR, and LT $\beta$ R protein expression was studied by immunohistochemistry in a cohort of patients, the vast majority of whom had undergone curative resection of a lung tumor in the University Hospital of Patras between 2005 and 2010 (Table 4). Selection of patients was serially and retrospectively done while patient data analysis was performed blindly based on the archive and database of the Pathology Department of the University Hospital of Patras. Invasive, formalin-fixed paraffin-embedded (FFPE) NSCLC tissue specimens as well as adjacent nonneoplastic lung parenchyma were retrieved.

Mouse monoclonal antibodies against CD40 and BAFFR and a rabbit polyclonal antibody against LT $\beta \mathrm{R}$ were used for antigen detection. Specific conditions regarding clonality, clone, dilution, antigen retrieval, and incubation time have been described in depth in previous publications (6); thus, here they are presented briefly (Table 5). Detection and visualization were performed using the EnVision Detection Kit (DAKO) and diaminobenzidine (DAB) chromogen following the manufacturer's instructions. In addition, dehydrated Harris' hematoxylin was used to counterstain the sections.

\section{Evaluation of Immunohistochemistry}

An evaluation of immunohistochemical staining has been described in detail in a previous publication (6). Briefly, an experienced pathologist (VT) assessed and scored each slide in a blind fashion. Tumor as well as stromal cells (myofibroblasts), tumor-infiltrating lymphocytes (TILs), and tumor-associated macrophages (TAMs) were scored. An initial selection of representative areas was performed at low $(\times 100)$ magnification. A minimum number of 1,000 cells per tissue section was counted at a $\times 400$ magnification. Cytoplasmic staining was evaluated for both epithelial and stromal cells, while membranous staining was performed only for epithelial cells. Microphotographs were taken by using a Nikon DXM1200C digital camera mounted on a Nikon Eclipse 80i microscope and ACT-1C software (Nikon Instruments Inc., Melville, NY, USA).

\section{Statistical Analysis}

Statistical analysis was performed by using Statistical Package for Social Sciences version 17 (SPSS, Chicago, IL, USA). Categorical nominal variables were evaluated using the chi-square test or Fisher's exact test. The T test was used for continuous variables with normal distribution. Analysis by using the Kruskal-Wallis or Mann-Whitney test was performed for ordinal or continuous data. Spearman's correlations were used to assess associations between variables. The Kaplan-Meier method and the log-rank test were used for plotting of survival rates and their comparison, respectively. $\mathrm{p}<0.05$ was considered statistically significant for all comparisons.

\section{RESULTS}

\section{Frequencies of Genotypes and Alleles Across Subpopulations}

In the current study, genotyping of the three studied polymorphisms was achieved in the vast majority of patients and controls, who were enrolled in the study. In particular, genotyping for CD40 rs1883832 (T>C) was successfully performed in 228 patients and all healthy controls (Table 6). The frequencies of the three CD40 rs1883832 genotypes (TT, CT, CC) were $23.6 \%, 26.8 \%$, and $49.6 \%$, in the NSCLC cases and $24.4 \%, 39.5 \%$, and $36.1 \%$ in healthy controls, respectively. In addition, BAFFR rs7290134 $(A>G)$ was successfully detected in all patients and 298 healthy controls. The frequencies of the three BAFFR rs7290134 (A>G) genotypes (AA, AG, GG) were $64.2 \%, 31.9 \%$, and $3.9 \%$ in lung cancer patients and $62.4 \%, 3.9 \%$, and $5.7 \%$ in healthy controls, respectively (Table 7). Furthermore, 223 NSCLC patients and all healthy controls were genotyped for $L T \beta R$ rs10849448 $(A>G)$. The frequencies for the three genotypes $\mathrm{AA}, \mathrm{AG}$, and GG of $L T \beta R$ rs10849448 (A>G) were $10.8 \%$, $27.4 \%$, and $61.8 \%$ in the NSCLC subcohort and $18.4 \%, 25.8 \%$, and $55.8 \%$ in healthy controls, respectively (Table 8).

\section{CD40 rs1883832 (T>C) and LT $\beta R$ rs10849448 (A>G) SNPs Were Associated With Increased NSCLC Risk}

As shown in Table 9, a statistically significant difference was observed in the allele frequency of CD40 rs1883832 (T) 
TABLE 4 | Clinicopathological characteristics and survival data of NSCLC patients studied by immunohistochemistry.

\begin{tabular}{|c|c|}
\hline Clinicopathological characteristics & Total cases $n(\%)$ \\
\hline Total & $96(100)$ \\
\hline Age (years) Median (range) & $67(44-84)$ \\
\hline \multicolumn{2}{|l|}{ Gender } \\
\hline Total & $96(100)$ \\
\hline Male & $90(93.8)$ \\
\hline Female & $6(6.2)$ \\
\hline \multicolumn{2}{|l|}{ Smoking (pack-years) } \\
\hline Total & $96(100)$ \\
\hline Cases (\%) & $39(40.6)$ \\
\hline Mean (range) & $90.92(20-165)$ \\
\hline NA & $57(59.4)$ \\
\hline \multicolumn{2}{|l|}{ Primary location } \\
\hline Total & $96(100)$ \\
\hline Left lung & 38 (39.6) \\
\hline Right lung & $58(60.4)$ \\
\hline NA & - \\
\hline \multicolumn{2}{|l|}{ Histology } \\
\hline Total & $96(100)$ \\
\hline Squamous & $56(58.3)$ \\
\hline Adenocarcinoma & 32 (33.3) \\
\hline Large carcinoma & $8(8.3)$ \\
\hline NA & - \\
\hline \multicolumn{2}{|l|}{ Stage } \\
\hline Total & $96(100)$ \\
\hline । & $33(34.4)$ \\
\hline$\|$ & $31(32.3)$ \\
\hline III & 30 (31.3) \\
\hline IV & $2(2.1)$ \\
\hline NA & - \\
\hline \multicolumn{2}{|l|}{ Grade } \\
\hline Total & $96(100)$ \\
\hline I & $4(4.2)$ \\
\hline$\|$ & $42(43.8)$ \\
\hline III & $42(43.8)$ \\
\hline NA & $8(8.3)$ \\
\hline \multicolumn{2}{|l|}{ Maximum diameter $(\mathrm{cm})$} \\
\hline Total & $96(100)$ \\
\hline Cases (\%) & $94(98.0)$ \\
\hline Mean (range) & $5.34(1.10-21.00)$ \\
\hline NA & $2(2.0)$ \\
\hline \multicolumn{2}{|l|}{ Lymph node infiltration } \\
\hline Total & 96 (100) \\
\hline No & $49(51.0)$ \\
\hline Yes & $43(45.8)$ \\
\hline NA & $3(3.1)$ \\
\hline \multicolumn{2}{|l|}{ Metastasis (adrenals) } \\
\hline Total & $96(100)$ \\
\hline No & $17(17.7)$ \\
\hline Yes & $1(1.0)$ \\
\hline NA & 78 (81.3) \\
\hline \multicolumn{2}{|l|}{ Metastasis (liver) } \\
\hline Total & $96(100)$ \\
\hline No & $17(17.7)$ \\
\hline Yes & $3(3.1)$ \\
\hline NA & $76(79.2)$ \\
\hline \multicolumn{2}{|l|}{ Metastasis (brain) } \\
\hline Total & $96(100)$ \\
\hline No & $17(17.7)$ \\
\hline Yes & $6(6.3)$ \\
\hline NA & $73(76.0)$ \\
\hline
\end{tabular}

TABLE 4 | Continued

Clinicopathological characteristics

Total cases $n(\%)$

Metastasis (bones)

Total

No

Yes

NA

Metastasis (adrenals-liver-brain-bones)

Total

No

Yes

NA

Survival (2 years)

Total

Dead

Alive

NA

Survival (3 years)

Total

Dead

Alive

NA

Survival (5 years)

Total

Dead

Alive

NA

Relapse

Total

No

Yes

NA

96 (100)

13 (13.5)

11 (11.5)

72 (75.0)

96 (100)

$6(6.3)$

21 (21.9)

69 (71.9)

96 (100)

37 (38.5)

57 (59.4)

2 (2.1)

96 (100)

50 (52.1)

42 (43.8)

4 (4.2)

96 (100)

59 (61.5)

33 (34.4)

4 (4.2)

96 (100)

8 (8.3)

14 (14.6)

74 (77.1)

NA, data not available or unknown.

between lung cancer patients and controls $(p=0.021)$ as well as between the frequencies of the studied genotypes $(p=0.003)$. Statistically significant was also the difference between genotypes in NSCLC patients and healthy controls following the dominant model for the $\mathrm{T}$ allele $(\mathrm{p}=0.002)$. In univariate analysis, $\mathrm{T}$ allele carriers (TT+CT) for CD40 rs1883832 had a higher risk for lung cancer $(\mathrm{p}=0.002$; OR $1.738,95 \% \mathrm{CI}$ 1.224-2.468). The same relation remained statistically significant under a multivariate model, in which age, smoking history, and genotype for CD40 rs1883832 SNP were entered as covariates ( $\mathrm{p}=0.007$; OR 1.701, 95\% CI 1.154-2.506). In addition, using an overdominant gene model (CT vs. CC+ TT), the CT genotype was also associated with increased risk for NSCLC not only in univariate analysis ( $\mathrm{p}=$ 0.002; OR 1.785, 95\% CI 1.228-2.594) but also after adjusting for cofactors $(\mathrm{p}=0.024$; OR 1.606, 95\% CI 1.065-2.422).

In addition, $L T \beta R$ rs10849448 (A>G) SNP was also associated with the development of NSCLC. Particularly, homozygosity for the alternative allele A was related to higher risk for NSCLC in univariate $(\mathrm{p}=0.016 ; \mathrm{OR}, 1.869 ; 95 \% 1.117-3.127)$ and multivariate analyses using age as cofactor $(\mathrm{p}=0.008$; OR, 2.106; 95\% 1.210-3.667). On the contrary, no association was observed between BAFFR rs7290134 SNP and the risk for NSCLC following any model. 
TABLE 5 | Primary antibodies and their clonality, clone, dilution, antigen retrieval, and incubation time information.

\begin{tabular}{|c|c|c|c|c|c|c|c|}
\hline Antibody & Clonality & Company & Catalogue number & Clone & Dilution & Antigen retrieval conditions & Incubation time \\
\hline LT $\beta R$ & $\mathrm{P}$ & Abcam & Ab193449 & & $1: 750$ & $8 \mathrm{mM}$ sodium citrate, $\mathrm{pH} 6.0$ & Overnight $4^{\circ} \mathrm{C}$ \\
\hline CD40 & M & SANTA CRUZ & Sc-13528 & LOB-11 & $1: 20$ & 1.2 mM EDTA, pH 8.0 & Overnight $4^{\circ} \mathrm{C}$ \\
\hline BAFFR & $M$ & SANTA CRUZ & Sc-32774 & $11 \mathrm{C} 1$ & $1: 20$ & 1.2 mM EDTA, pH 8.0 & Overnight $4^{\circ} \mathrm{C}$ \\
\hline
\end{tabular}

TABLE 6 | Relationships between clinicopathological variables of NSCLC patients and CD40 rs1883832 genotypes.

\begin{tabular}{|c|c|c|c|c|c|c|c|}
\hline \multirow[t]{2}{*}{ Clinicopathological characteristics } & \multirow{2}{*}{$\begin{array}{c}\text { Patients } \\
\text { n (\%) }\end{array}$} & \multicolumn{2}{|c|}{ Genotypes } & \multirow[t]{2}{*}{ p-value } & \multicolumn{2}{|c|}{ Genotypes } & \multirow[t]{2}{*}{ p-value } \\
\hline & & cC & $\mathrm{CT}+\mathrm{TT}$ & & TT & $\mathrm{CC}+\mathrm{CT}$ & \\
\hline Total & $229(100)$ & & & & & & \\
\hline Genotyped & 228 (99.6) & $113(49.3)$ & $115(50.2)$ & & $54(23.6)$ & $174(76.0)$ & \\
\hline NA & $1(0.4)$ & & & & & & \\
\hline Age (years) Mean (range) & $65(40-84)$ & & & & & & \\
\hline Genotyped & $228(100)$ & $39(17.1)$ & $74(32.5)$ & 0.584 & $22(9.6)$ & $61(26.8)$ & 0.518 \\
\hline$<65$ & $83(36.4)$ & 44 (19.3) & $71(31.3)$ & & $32(14.0)$ & $113(49.6)$ & \\
\hline$>=65$ & $145(63.6)$ & & & & & & \\
\hline \multicolumn{8}{|l|}{ Gender } \\
\hline Genotyped & $228(100)$ & $101(44.3)$ & $106(46.5)$ & 0.500 & $50(21.9)$ & $157(68.9)$ & 0.789 \\
\hline Male & $207(90.8)$ & $12(5.3)$ & 9 (3.9) & & $4(1.8)$ & $17(7.5)$ & \\
\hline Female & $21(9.2)$ & & & & & & \\
\hline \multicolumn{8}{|l|}{ Smoking (pack-years) } \\
\hline Genotyped & $228(100)$ & $48(21.1)$ & $41(18.0)$ & 0.840 & $21(9.2)$ & $68(29.8)$ & 0.927 \\
\hline Cases & 89 (39.0) & $85.18(10-165)$ & $90.30(15-200)$ & & $88.19(15-200)$ & $87.34(10-180)$ & \\
\hline Mean (range) & $87.12(10-200)$ & $65(28.5)$ & $74(32.5)$ & & $33(14.5)$ & $106(46.5)$ & \\
\hline$v \alpha$ & $139(61.0)$ & & & & & & \\
\hline \multicolumn{8}{|l|}{ Primary location } \\
\hline Genotyped & $228(100)$ & 46 & 54 & 0.343 & 23 & 77 & 1.000 \\
\hline Left lung & $100(43.9)$ & 64 & 56 & & 28 & 92 & \\
\hline Right lung & $120(52.6)$ & $3(1.3)$ & $5(2.2)$ & & $3(1.3)$ & $5(2.2)$ & \\
\hline NA & $8(3.5)$ & & & & & & \\
\hline Histology & & & & & & & 0.958 \\
\hline Genotyped & $228(100)$ & 51 & 60 & 0.572 & 27 & 84 & \\
\hline Squamous & 111 & 52 & 46 & & 23 & 75 & \\
\hline Adenocarcinoma & 98 & 5 & 6 & & 3 & 8 & \\
\hline Large carcinoma & 11 & $5(2.2)$ & $3(1.3)$ & & $1(0.4)$ & $7(3.1)$ & \\
\hline NA & $8(3.5)$ & & & & & & \\
\hline \multicolumn{8}{|l|}{ Stage } \\
\hline Genotyped & $228(100)$ & 27 & 31 & 0.226 & 13 & 45 & 0.754 \\
\hline I & 58 & 30 & 20 & & 10 & 40 & \\
\hline$\|$ & 50 & 35 & 34 & & 19 & 50 & \\
\hline III & 69 & 15 & 24 & & 8 & 31 & \\
\hline IV & 39 & $6(2.6)$ & $6(2.6)$ & & $4(1.8)$ & $8(3.5)$ & \\
\hline NA & $12(5.3)$ & & & & & & \\
\hline \multicolumn{8}{|l|}{ Grade } \\
\hline Genotyped & $228(100)$ & 4 & 2 & 0.570 & 0 & 6 & 0.262 \\
\hline 1 & 6 & 41 & 44 & & 23 & 62 & \\
\hline$\|$ & 85 & 48 & 41 & & 19 & 70 & \\
\hline III & 89 & $20(8.8)$ & $28(12.3)$ & & $12(5.3)$ & $36(15.8)$ & \\
\hline NA & $48(21.1)$ & & & & & & \\
\hline \multicolumn{8}{|l|}{ Maximum diameter $(\mathrm{cm})$} \\
\hline Genotyped & $228(100)$ & 95 & 91 & 0.435 & 44 & 142 & 0.618 \\
\hline Cases (\%) & 186 & $5.02(1.20-14)$ & $4.68(0.7-21)$ & & $4.8(0.7-21)$ & $4.88(1.1-14)$ & \\
\hline Mean (range) & $4.87(0.7-21)$ & $18(7.9)$ & $24(10.5)$ & & $10(4.4)$ & $32(14.0)$ & \\
\hline NA & $42(18.4)$ & & & & & & \\
\hline \multicolumn{8}{|l|}{ Lymph node infiltration } \\
\hline Genotyped & $228(100)$ & 43 & 48 & 0.286 & 25 & 66 & 0.287 \\
\hline No & 91 & 44 & 35 & & 16 & 63 & \\
\hline Yes & 79 & $26(11.4)$ & $32(14.0)$ & & $13(5.7)$ & $45(19.7)$ & \\
\hline NA & $58(25.4)$ & & & & & & \\
\hline \multicolumn{8}{|l|}{ Metastasis (adrenals) ${ }^{a}$} \\
\hline Genotyped & $228(100)$ & 18 & 17 & 0.384 & 8 & 27 & 0.730 \\
\hline
\end{tabular}


TABLE 6 | Continued

\begin{tabular}{|c|c|c|c|c|c|c|c|}
\hline Clinicopathological characteristics & $\begin{array}{c}\text { Patients } \\
\text { n (\%) }\end{array}$ & \multicolumn{2}{|c|}{ Genotypes } & $p$-value & \multicolumn{2}{|c|}{ Genotypes } & $p$-value \\
\hline No & 35 & 6 & 10 & & 5 & 11 & \\
\hline NA & $177(77.6)$ & & & & & & \\
\hline \multicolumn{8}{|l|}{ Metastasis (liver) ${ }^{a}$} \\
\hline Genotyped & $228(100)$ & & & & & & \\
\hline NA & $175(76.8)$ & $8(38.6)$ & $87(38.2)$ & & $4(18.0)$ & $134(58.8)$ & \\
\hline \multicolumn{8}{|l|}{ Metastasis (brain) ${ }^{a}$} \\
\hline Genotyped & $228(100)$ & 19 & 15 & 0.144 & 8 & 26 & 0.784 \\
\hline No & 34 & 12 & 21 & & 9 & 24 & \\
\hline Yes & 33 & $82(36.0)$ & 79 (34.6) & & $37(16.2)$ & $124(54.4)$ & \\
\hline NA & $161(70.6)$ & & & & & & \\
\hline \multicolumn{8}{|l|}{ Metastasis (adrenals-liver-brain-bones) ${ }^{a}$} \\
\hline Genotyped & $228(100)$ & 10 & 3 & 0.032 & 1 & 12 & 0.283 \\
\hline No & 13 & 30 & 43 & & 19 & 54 & \\
\hline Yes & 73 & & & & & & \\
\hline \multicolumn{8}{|l|}{ NA } \\
\hline \multicolumn{8}{|l|}{ Survival (2 years) } \\
\hline Genotyped & $228(100)$ & 50 & 55 & 0.687 & 19 & 86 & 0.081 \\
\hline Dead & 105 & 59 & 57 & & 33 & 83 & \\
\hline Alive & 116 & $4(1.8)$ & $3(1.3)$ & & $2(0.9)$ & $5(2.2)$ & \\
\hline NA & $7(3.1)$ & & & & & & \\
\hline \multicolumn{8}{|l|}{ Survival (3 years) } \\
\hline Genotyped & $228(100)$ & 60 & 74 & 0.166 & 25 & 109 & 0.033 \\
\hline
\end{tabular}

NA, data not available or unknown.

${ }^{a}$ Metastasis detected at follow-up, not at the time of sample collection.

TABLE 7 | Relationships between clinicopathological variables of NSCLC patients and BAFFR rs7290134 genotypes.

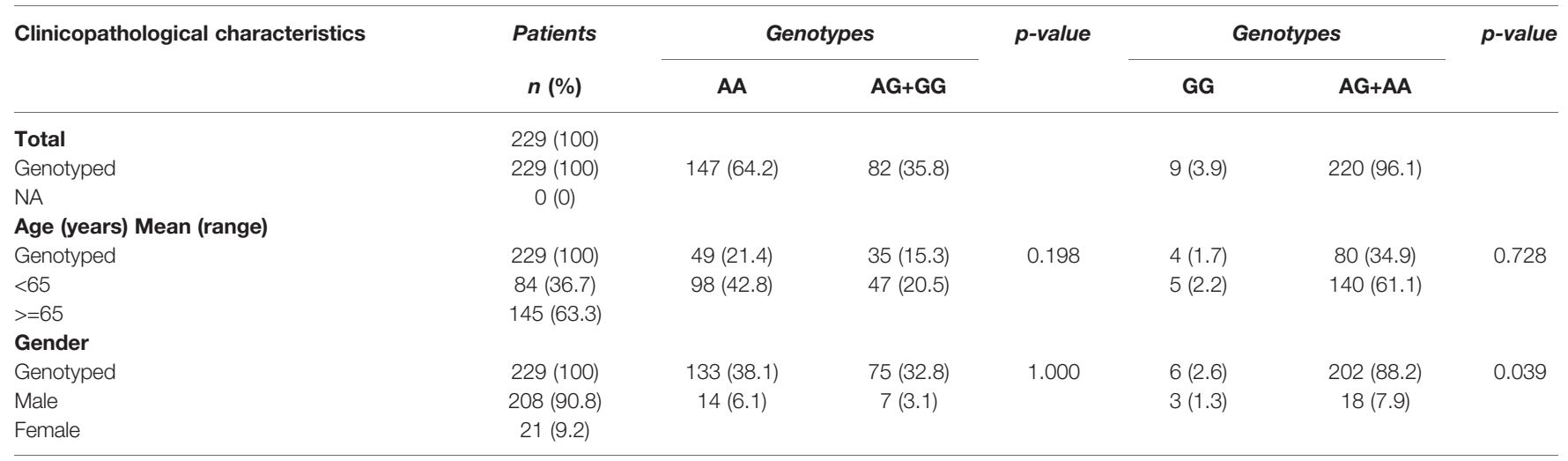


TABLE 7 | Continued

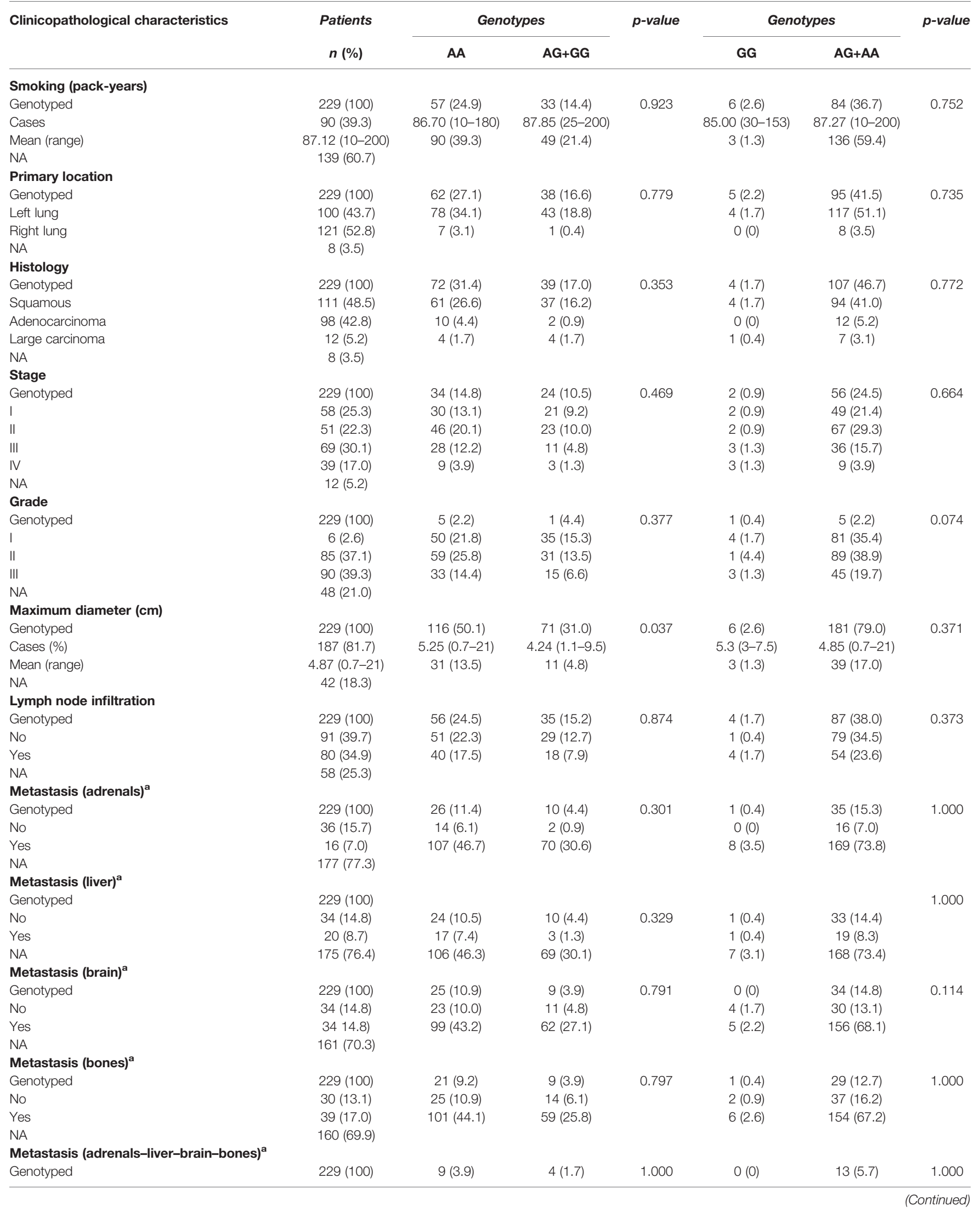


TABLE 7 | Continued

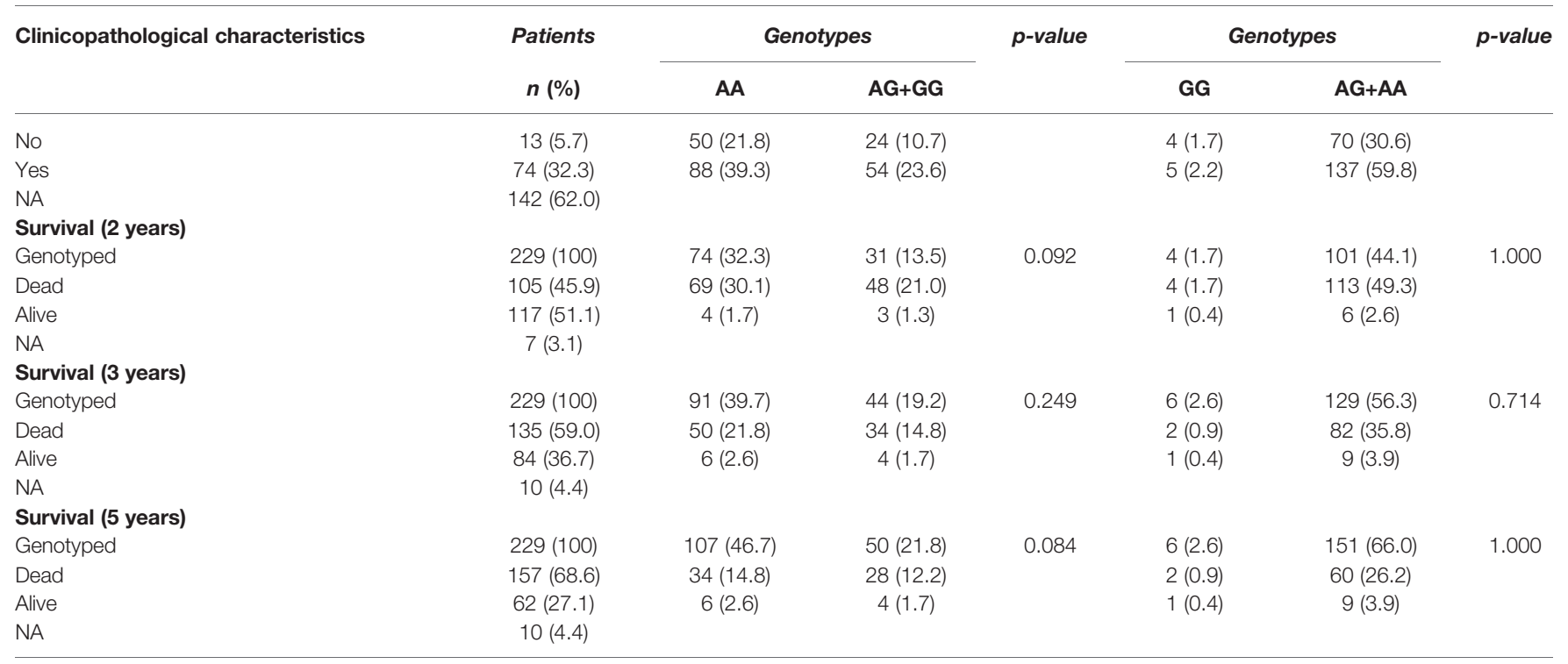

NA, data not available or unknown.

${ }^{a}$ Metastasis detected at follow-up, not at the time of sample collection.

TABLE 8 | Relationships between clinicopathological variables of NSCLC patients and LtBR rs10849448 genotypes.

\begin{tabular}{|c|c|c|c|c|c|c|c|}
\hline Clinicopathological characteristics & $\begin{array}{l}\text { Patients } \\
\text { n (\%) }\end{array}$ & \multicolumn{2}{|c|}{ Genotypes } & $p$-value & \multicolumn{2}{|c|}{ Genotypes } & $p$-value \\
\hline Total & 229 (100) & & & & & & \\
\hline NA & $6(2.6)$ & & & & & & \\
\hline Age (years) Mean (range) & $65(40-84)$ & & & & & & \\
\hline Genotyped & $223(100)$ & $44(19.7)$ & 37 (16.6) & 0.087 & $9(4.0)$ & $72(32.3)$ & 1.000 \\
\hline \multicolumn{8}{|l|}{ Gender } \\
\hline Genotyped & $223(100)$ & $126(56.5)$ & 77 (34.5) & 1.000 & $22(9.9)$ & $181(81.2)$ & 1.000 \\
\hline Male & $203(91.0)$ & $12(5.4)$ & $8(3.6)$ & & $2(0.9)$ & $18(8.1)$ & \\
\hline Female & $20(9.0)$ & & & & & & \\
\hline \multicolumn{8}{|l|}{ Smoking (pack-years) } \\
\hline Genotyped & $223(100)$ & $51(22.7)$ & $35(15.7)$ & 0.748 & $11(4.9)$ & $75(33.6)$ & 0.564 \\
\hline Left lung & $97(43.5)$ & $73(32.7)$ & $45(20.2)$ & & $13(5.8)$ & $105(47.1)$ & \\
\hline Right lung & $118(52.9)$ & $6(2.7)$ & $2(0.9)$ & & $1(0.4)$ & $7(3.1)$ & \\
\hline NA & $8(3.6)$ & & & & & & \\
\hline \multicolumn{8}{|l|}{ Histology } \\
\hline Genotyped & $223(100)$ & $73(32.7)$ & $35(15.7)$ & 0.202 & $6(2.7)$ & $102(45.7)$ & 0.065 \\
\hline Squamous & $108(48.4)$ & $55(24.7)$ & $42(18.8)$ & & $15(6.7)$ & $82(36.8)$ & \\
\hline Adenocarcinoma & $97(43.5)$ & $5(2.2)$ & $5(2.2)$ & & $1(0.4)$ & $9(4.0)$ & \\
\hline Large carcinoma & $10(4.5)$ & $5(2.2)$ & $3(1.3)$ & & $2(0.9)$ & $6(2.7)$ & \\
\hline NA & $8(3.6)$ & & & & & & \\
\hline \multicolumn{8}{|l|}{ Stage } \\
\hline Genotyped & $223(100)$ & $31(13.9)$ & $24(10.8)$ & 0.631 & $6(2.7)$ & $49(22.0)$ & 0.216 \\
\hline I & $55(24.7)$ & $28(12.6)$ & $21(9.4)$ & & $7(3.1)$ & $42(18.8)$ & \\
\hline
\end{tabular}


TABLE 8 | Continued

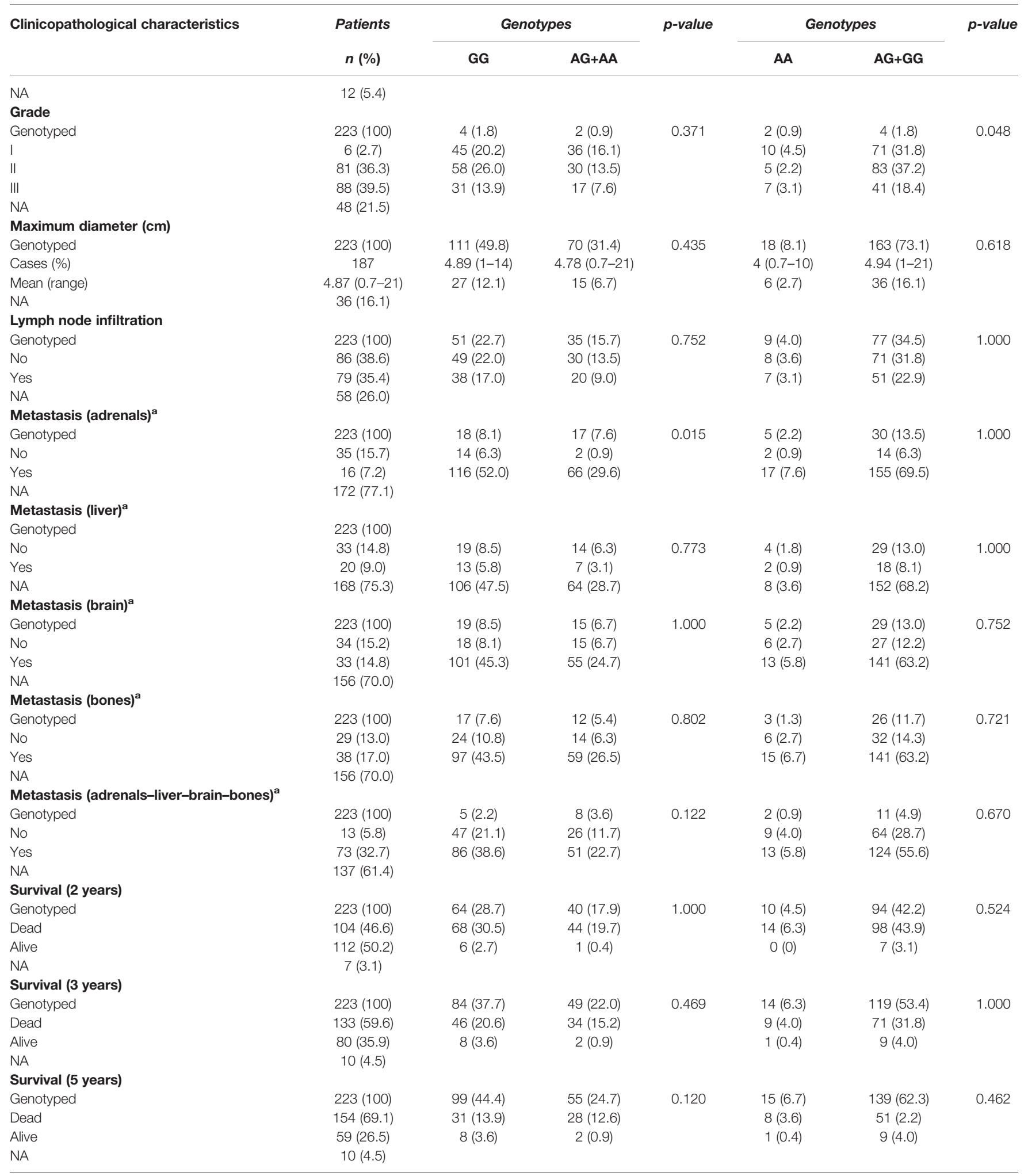

NA, data not available or unknown.

${ }^{a}$ Metastasis detected at follow-up, not at the time of sample collection. 
TABLE 9 | ORs and 95\% Cls for NSCLC in relation to genotypes of studied SNPs.

\begin{tabular}{|c|c|c|c|c|c|c|c|}
\hline Genotype & Cases n (\%) & Controls n (\%) & $p^{a}$ & ${ }^{b}$ Crude OR (95\% Cl) & $p$ & ${ }^{\mathrm{c}}$ Adjusted OR (95\% CI) & $p$ \\
\hline Total & 229 & 299 & - & - & - & - & - \\
\hline$C D 40$ rs1883832 (T>C) & 228 & 299 & - & - & - & - & - \\
\hline$\pi$ & $54(23.7)$ & $73(24.4)$ & 0.003 & $1.414(0.911-2.196)$ & 0.122 & $1.508(0.930-2.445)$ & 0.096 \\
\hline CT & $61(26.8)$ & $118(39.5)$ & & 2.024 (1.348-3.039) & 0.001 & 1.865 (1.192-2.919) & 0.006 \\
\hline $\mathrm{CC}$ & $113(49.5)$ & $108(36.1)$ & & 1.000 & - & 1.000 & - \\
\hline $\mathrm{TT}+\mathrm{CT}$ & $115(50.4)$ & $191(63.9)$ & 0.002 & 1.738 (1.224-2.468) & 0.002 & 1.701 (1.154-2.506) & 0.007 \\
\hline vs. CC & $113(49.6)$ & $108(36.1)$ & & & & & \\
\hline$\pi$ & $54(23.7)$ & $73(24.4)$ & 0.846 & $1.041(0.695-1.558)$ & 0.846 & $1.156(0.742-1.800)$ & 0.523 \\
\hline vs. $\mathrm{CT}+\mathrm{CC}$ & $174(76.3)$ & $226(75.6)$ & & & & & \\
\hline CT & $61(26.8)$ & $118(39.5)$ & 0.002 & 1.785 (1.228-2.594) & 0.002 & 1.606 (1.065-2.422) & 0.024 \\
\hline vs. TT+CC & $167(73.2)$ & $181(60.5)$ & & & & & \\
\hline T allele & $169(37.1)$ & $264(44.1)$ & 0.021 & 1.342 (1.046-1.722) & 0.021 & $1.384(1.052-1.821)$ & 0.020 \\
\hline C allele & 287 (62.9) & $334(55.9)$ & & $0.745(0.581-0.956)$ & 0.021 & $0.723(0.549-0.951)$ & 0.020 \\
\hline BAFFR rs7290134 (A>G) & 229 & 298 & - & - & - & - & - \\
\hline AA & $147(64.2)$ & $186(62.4)$ & 0.640 & 1.000 & - & 1.000 & - \\
\hline$A G$ & 73 (31.9) & 95 (31.9) & & $1.029(0.708-1.495)$ & 0.883 & $1.054(0.697-1.593) 0$ & 0.803 \\
\hline GG & $9(3.9)$ & $17(5.7)$ & & $1.493(0.647-3.446)$ & 0.348 & 1.647 (0.669-4.056) & 0.278 \\
\hline AA vs & $147(64.2)$ & $186(62.4)$ & 0.675 & $0.926(0.648-1.325)$ & 0.675 & $0.894(0.603-1.326)$ & 0.578 \\
\hline$A G+G G$ & $82(35.8)$ & $112(37.6)$ & & & & & \\
\hline$A A+A G$ & $220(96.1)$ & $281(94.3)$ & 0.351 & $0.676(0.296-1.546)$ & 0.354 & $0.666(0.271-1.637)$ & 0.376 \\
\hline vs. GG & $9(3.9)$ & $17(5.7)$ & & & & & \\
\hline AG vs. & $73(31.9)$ & $96(32.1)$ & 0.955 & $1.011(0.699-1.462)$ & 0.955 & $1.039(0.691-1.561)$ & 0.854 \\
\hline $\mathrm{AA}+\mathrm{GG}$ & $156(68.1)$ & $203(67.9)$ & & & & & \\
\hline A allele & $367(80.1)$ & 467 (78.4) & 0.482 & $0.898(0.664-1.213)$ & 0.482 & $0.864(0.621-1.202)$ & 0.385 \\
\hline G allele & 91 (19.9) & $129(21.6)$ & & $1.114(0.492-0.929)$ & 0.482 & $1.158(0.832-1.611)$ & 0.385 \\
\hline$L T \beta R$ rs10849448 (A>G) & 223 & 299 & - & - & - & - & - \\
\hline $\mathrm{AA}$ & $24(10.8)$ & 55 (18.4) & 0.054 & $1.894(1.115-3.217)$ & 0.018 & 2.051 (1.159-3.628) & 0.014 \\
\hline$A G$ & $61(27.4)$ & $77(25.8)$ & & $1.043(0.696-1.563)$ & 0.838 & $0.934(0.593-1.473)$ & 0.934 \\
\hline GG & $138(61.8)$ & $167(55.8)$ & & 1.000 & - & 1.000 & - \\
\hline AA vs & $24(10.8)$ & $55(18.4)$ & 0.016 & 1.869 (1.117-3.127) & 0.017 & 2.106 (1.210-3.667) & 0.008 \\
\hline$A G+G G$ & $199(89.2)$ & $244(81.6)$ & & & & & \\
\hline$A A+A G$ vs & $85(38.1)$ & $132(44.1)$ & 0.167 & $0.779(0.547-1.110)$ & 0.167 & $1.255(0.848-1.856)$ & 0.256 \\
\hline GG & $138(61.9)$ & 167 (55.9) & & & & & \\
\hline$A G$ & $61(27.4)$ & 77 (25.8) & 0.681 & $0.921(0.622-1.364)$ & 0.681 & 0.995 (0.978-1.013) & 0.602 \\
\hline vs, GG+AA & $162(72.6)$ & $222(74.2)$ & & & & & \\
\hline A allele & $109(24.4)$ & $187(31.3)$ & 0.015 & 1.407 (1.067-1.855) & 0.016 & $1.436(1.060-1.945)$ & 0.019 \\
\hline G allele & 337 (75.6) & $411(68.7)$ & & $0.711(0.539-0.937)$ & 0.016 & $0.696(0.514-0.943)$ & 0.019 \\
\hline
\end{tabular}

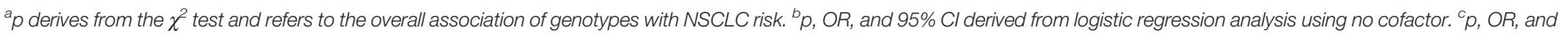
95\% $\mathrm{Cl}$ derived from logistic regression analysis using age and gender as cofactors.

$\mathrm{Cl}$, confidence interval; OR, odds ratio.

Bold text indicates a statistically significant correlation with a p-value less than 0.05 .

\section{CD40 rs1883832 (T>C) Was Associated With Overall Survival}

Among all NSCLC cases, the CD40 rs1883832 CT heterozygotes had poorer OS compared to TT and CC homozygotes after 2,3 , and 5 years of observation on univariate analysis $(\mathrm{p}=0.015, \mathrm{p}=0.005$, and $\mathrm{p}=0.017$, respectively, Figure 1). Prognostic significance for 2-, 3-, and 5 -year OS persisted in multivariate analyses adjusted for age, sex, stage, and histological subtypes $(\mathrm{p}=0.023, \mathrm{p}<0.001$, and $\mathrm{p}=0.001$, respectively). The same correlation was also observed after stratifying with histological subtype $(\mathrm{p}=0.075$ and $\mathrm{p}=0.001$, respectively). Interestingly, further analysis by stage stratification revealed that the observed correlation was limited in stages I and II ( $\mathrm{p}=0.001)$, while it disappeared in patients of stages III and IV $(\mathrm{p}=0.151)$.

On the contrary, although BAFFR rs7290134 (A>G) SNP seemed to be associated with the OS, finally it did not reach the level of statistical significance $(\mathrm{p}=0.087)$. In particular, $\mathrm{G}$ allele carriers had better 5-year survival compared to AA homozygotes; however, this difference was not statistically significant.

\section{$C D 40$ rs1883832 (T>C), LT $\beta R$ rs10849448 $(A>G)$, and BAFFR rs7290134 (A>G) Were Associated With Protein Expression}

CD40 rs1883832 was associated with CD40 expression. In particular, CD40 rs1883832 was associated with total (cytoplasmic and membranous) CD40 expression with CC homozygotes having higher tumorous CD40 (Mann-Whitney $\mathrm{U}$ test, $\mathrm{p}=0.040$ ). In addition, $\mathrm{CC}$ homozygotes had higher CD40 expression in stromal cells compared to $\mathrm{T}$ allele carriers (MannWhitney $\mathrm{U}$ test, $\mathrm{p}=0.036$ ). In addition, $L T \beta R$ rs10849448 SNP was associated with LT $\beta$ R membranous expression, with AA homozygotes having higher protein levels $(\mathrm{p}=0.035)$. Furthermore, the third SNP, BAFFR rs7290134 (A>G), was associated with BAFFR membranous expression with A minor allele carriers having lower protein levels $(\mathrm{p}=0.039)$. 

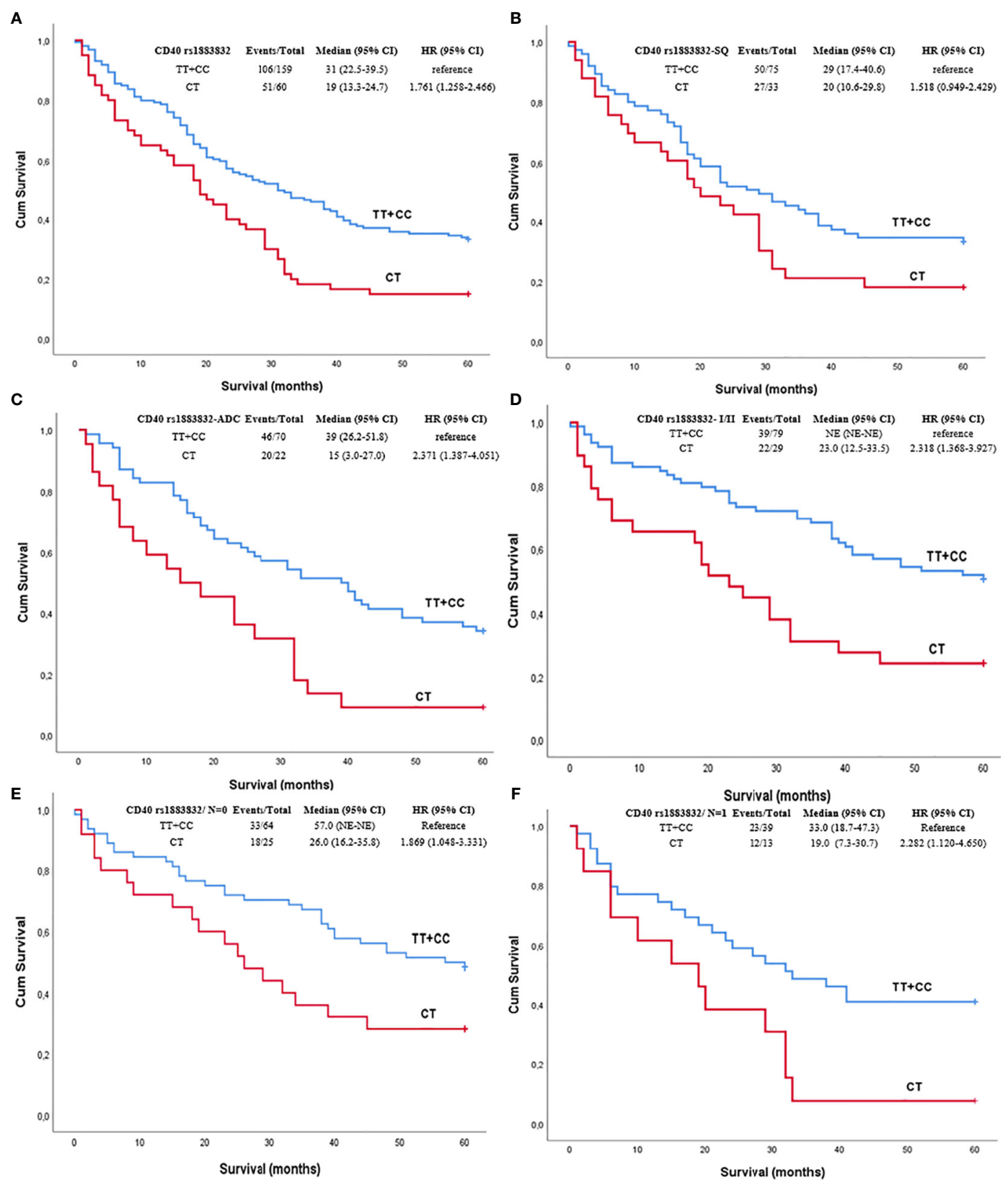

FIGURE 1 | Kaplan-Meier curves depicting overall survival (OS) of NSCLC patients in relation to (A) CD40 rs1883832 genotypes in the whole sub-cohort of NSCLC patients, (B) CD40 rs1883832 genotypes in SQ patients, (C) CD40 rs1883832 genotypes in ADC patients, (D) CD40 rs1883832 genotypes in stage I and II patients, (E) CD40 rs1883832 genotypes in NSCLC patients with $\mathrm{N}=0$, (F) CD40 rs1883832 genotypes in NSCLC patients with $\mathrm{N}=1$. SQ, squamous cell carcinoma; $A D C$, adenocarcinoma.

\section{CD40 rs1883832 (T>C) SNP Was Associated With Development of Metastases}

CD40 rs1883832 was associated with development of metastases after the initial assessment. Patients with no development of metastatic disease were mainly CC homozygotes $(p=0.022)$. The same association was also observed in multivariate analysis using age, sex, lymph node infiltration, and histological subtype as cofactors $(\mathrm{p}=0.019)$.

\section{DISCUSSION}

During the last years, accumulating evidence supports the role of NF- $\kappa \mathrm{B}$ in the pathobiology and management of NSCLC (25). In this context, CD40, BAFFR, and LT $\beta$ R cell surface receptors, which mainly activate NF- $\kappa \beta$ pathways, also seem to be important for NSCLC. In this study, we assessed the clinical value of three SNPs CD40 (rs1883832), BAFFR (rs7290134), and 
$L T \beta R$ (rs10849448) in NSCLC, with the results further supporting previous findings from our and other groups on the role of these molecules in NSCLC. One of the major findings of the study was the association of CD40 rs1883832 with NSCLC risk. In line with our observation, Krishnappa et al. reported that the rs1883832 $\mathrm{T}$ allele is associated with increased susceptibility to cervical cancer in the Malaysian population (17). Similarly, Shuang et al. have also shown that the rs1883832 $\mathrm{T}$ allele is related to sporadic breast cancer risk in Han Chinese women (18). Interestingly, the same allele of rs1883832 (T) has also been documented in a small study ( $\mathrm{n}=105$ cases) and has been correlated with the susceptibility to lung cancer in the Chinese population including not only NSCLC but also SCLC patients (19). Furthermore, TT genotype has also been associated with an increased risk for follicular lymphoma (26).

Another intriguing finding of the present study was the association of CD40 rs1883832 with OS. Although the role of this SNP has been studied in many nonmalignant diseases, as mentioned in the introduction, there has not been yet any reported finding on its prognostic significance in solid tumors or hematological malignancies. However, some indirect results are supportive of our observation. Our group has shown that CD40 overexpression in NSCLC patients is associated with improved 5-year OS (6), a finding, which is confirmed by using publicly available data and the KM plotter (Figure 2A) (27). In addition, in the current study, we show that CD40 rs1883832 is associated with total CD40 expression in tumorous cells as well as in stromal cells, suggesting a possible mechanism through which CD40 rs1883832 may influence survival outcome of NSCLC patients. From a biological point of view, this association could be justified by the genomic position of rs1883832, since it is located on the promoter region and in particular at the -1 base from the start codon of the CD40 and within the Kozak sequence (28). Also supportive is the observation by Skibola et al. according to which follicular lymphoma patients and healthy controls with TT genotypes had decreased plasma circulating soluble CD40 as well as CD40 cell surface expression in dendritic cells from healthy individuals compared to CC homozygotes (26).

Our study also showed that $L T \beta R$ rs10849448 $(A>G)$ SNP is associated with NSCLC risk, with homozygosity for the alternative A allele being related to lower risk for NSCLC, a finding that correlates for the first time this variant with cancer risk. Additionally, our group has reported that $L T \beta R$ expression in NSCLC has prognostic significance (6). The prognostic significance of $L T \beta R$ expression has also been confirmed using the KM plotter and the 203005_at dataset, with higher expression being correlated with poorer outcome (Figure 2B). In line with these findings, we also observed that $L T \beta R$ rs10849448 $(A>G)$ SNP was associated with LT $\beta R$ membranous expression with AA homozygotes having higher protein levels, providing concurrently a potent explanation for the increased risk for NSCLC.

Despite the promising results of our study, we must acknowledge some weak points. A major limitation of our study is that the retrospectively collected samples as well as the
A

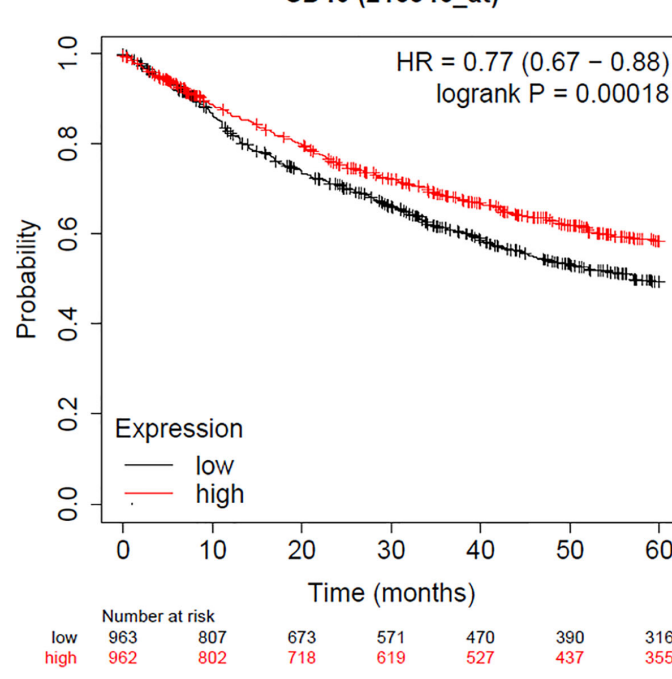

B

TNFCR (203005_at)

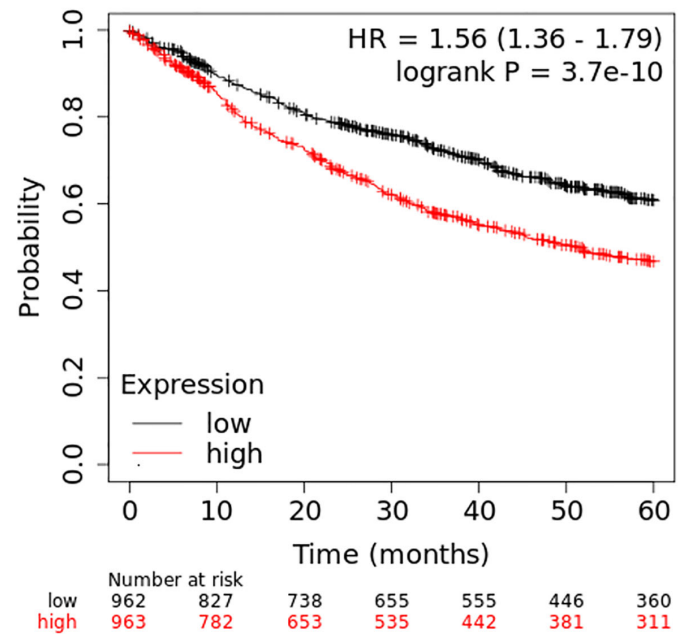

FIGURE 2 | (A) Kaplan-Meier curves depicting 5-year OS of NSCLC patients in relation to CD40 mRNA levels as provided by KM plotter, (B) Kaplan-Meier curves depicting 5-year OS of NSCLC patients in relation to LT $\beta R$ mRNA levels as provided by the KM plotter.

size of the cohort did not permit a separate analysis for the discovery and validation subgroups; thus, despite our initial intention to follow a two-phase design, the final analysis was based on the study of the whole cohort. A larger cohort could permit the separate analysis and lead to more robust results. Moreover, molecular profiling of driver mutations and PD-L1 status was not available since the patients were enrolled before immunotherapy era.

\section{CONCLUSIONS}

In conclusion, this study shows that two (CD40 rs1883832 and $L T \beta R$ rs 10849448) of the three studied genetic variants are associated with an increased risk for NSCLC, while CD40 
rs1883832 was also significantly associated with OS of patients with NSCLC. However, our findings need to be further validated in another population and more data are needed regarding the functionality of the studied polymorphisms.

\section{DATA AVAILABILITY STATEMENT}

The datasets from sequencing presented in this study can be found in online repository as well as are also available upon request from the corresponding author. The name of the repository and accession number can be found below: European Nucleotide Archive (ENA) (accession number: PRJEB47384).

\section{ETHICS STATEMENT}

This study was conducted according to the guidelines of the Declaration of Helsinki and was performed upon approval by the Scientific Committee and the Committee on Research and Ethics of the University Hospital of Patras (Greece, 22/18.2.2015).

\section{REFERENCES}

1. Dimitrakopoulos FD, Kottorou AE, Kalofonou M, Kalofonos HP. The Fire Within: NF-KappaB Involvement in Non-Small Cell Lung Cancer. Cancer Res (2020) 80(19):4025-36. doi: 10.1158/0008-5472.CAN-19-3578

2. Dimitrakopoulos FI, Antonacopoulou AG, Kottorou A, Vlotinou H, Panagopoulos ND, Dougenis D, et al. NSCLC and the Alternative Pathway of NF-Kappab: Uncovering an Unknown Relation. Virchows Archiv: Int J Pathol (2012) 460(5):515-23. doi: 10.1007/s00428-012-1230-2

3. Tang X, Liu D, Shishodia S, Ozburn N, Behrens C, Lee JJ, et al. Nuclear FactorKappaB (NF-Kappab) Is Frequently Expressed in Lung Cancer and Preneoplastic Lesions. Cancer (2006) 107(11):2637-46. doi: 10.1002/cncr.22315

4. Zhang Z, Ma J, Li N, Sun N, Wang C. Expression of Nuclear factor-kappaB and Its Clinical Significance in Nonsmall-Cell Lung Cancer. Ann Thoracic Surg (2006) 82(1):243-8. doi: 10.1016/j.athoracsur.2006.01.049

5. Dimitrakopoulos FD, Antonacopoulou AG, Kottorou AE, Panagopoulos N, Kalofonou F, Sampsonas F, et al. Expression Of Intracellular Components of the NF-KappaB Alternative Pathway (NF-Kappab2, RelB, NIK and Bcl3) Is Associated With Clinical Outcome of NSCLC Patients. Sci Rep (2019) 9 (1):14299. doi: 10.1038/s41598-019-50528-y

6. Dimitrakopoulos FD, Kottorou AE, Antonacopoulou AG, Panagopoulos N, Scopa C, Kalofonou M, et al. Expression of Immune System-Related Membrane Receptors CD40, RANK, BAFFR and LTbetaR Is Associated With Clinical Outcome of Operated Non-Small-Cell Lung Cancer Patients. J Clin Med (2019) 8(5):741. doi: 10.3390/jcm8050741

7. Sabel MS, Yamada M, Kawaguchi Y, Chen FA, Takita H, Bankert RB. CD40 Expression on Human Lung Cancer Correlates With Metastatic Spread. Cancer Immunol Immunother: CII (2000) 49(2):101-8. doi: 10.1007/ s002620050608

8. Ishikawa K, Miyamoto M, Yoshioka T, Kato T, Kaji M, Ohbuchi T, et al. UpRegulation of CD40 With Juxtacrine Activity in Human Nonsmall Lung Cancer Cells Correlates With Poor Prognosis. Cancer (2008) 113(3):530-41. doi: $10.1002 /$ cncr.23618

9. Dimtrakopoulos F-ID, Kottorou AE, Antonacopoulou AG, Nikolakopoulos A, Panagopoulos N, Kalofonou M, et al. Association of BAFFR Expression in CAFs With Overall Survival and Response to Platinum-Based Chemotherapy in NSCLC. J Clin Oncol (2019) 37(15_suppl):8537-. doi: 10.1200/JCO.2019.37.15_suppl.8537

10. Yun Y, Ma C, Ma X. The SNP Rs1883832 in CD40 Gene and Risk of Atherosclerosis in Chinese Population: A Meta-Analysis. PloS One (2014) 9 (5):e97289. doi: 10.1371/journal.pone.0097289
Written informed consent was obtained from all participants unless the Committee had granted a waiver.

\section{AUTHOR CONTRIBUTIONS}

Conceptualization, F-ID, AA, AK, and HK. Methodology, AA, AK, F-ID, and VT. Formal analysis, F-ID and AA. Investigation, F-ID, AA, AK, NP, DD, TM, AngK, and HK. Resources, F-ID and HK. Writing-original draft preparation, F-ID, AA, and AK. Writing-review and editing, F-ID, MK, and HK. All authors contributed to the article and approved the submitted version.

\section{FUNDING}

This research was cofunded by the Hellenic Society of Medical Oncology (HeSMO) through a research funding program as well as from EOGE, an Oncology Research Fund non-profit organization, in Greece.

11. Wang M, Li Y, Li W, Xia ZE, Wu Q. The CD40 Gene Polymorphism Rs1883832 Is Associated With Risk of Acute Coronary Syndrome in a Chinese Case-Control Study. DNA Cell Biol (2011) 30(3):173-8. doi: 10.1089/dna.2010.1129

12. Tian C, Qin W, Li L, Zheng W, Qiu F. A Common Polymorphism in CD40 Kozak Sequence $(-1 \mathrm{C} / \mathrm{T})$ Is Associated With Acute Coronary Syndrome. Biomed Pharmacother (2010) 64(3):191-4. doi: 10.1016/j.biopha.2009.09.021

13. Ma Y, Wang SX, Liu Y, Peng GG, Wang XM, Zhang B, et al. Single Nucleotide Polymorphism of CD40 in the 5'-Untranslated Region Is Associated With Ischemic Stroke. Gene (2013) 529(2):257-61. doi: 10.1016/ j.gene.2013.07.086

14. Liu Y, Liang WB, Gao LB, Wang YY, Zhang L. Association of CD40 -1c/T Polymorphism in the 5'-Untranslated Region and Chronic Obstructive Pulmonary Disease. Clin Chim Acta Int J Clin Chem (2009) 408(1-2):56-9. doi: 10.1016/j.cca.2009.07.005

15. Zhou C, Jin X, Tang J, Fei J, Gu C, Chen X. Association of CD40 -1c/T Polymorphism in the 5'-Untranslated Region With Chronic HBV Infection. Cell Physiol Biochem: Int J Exp Cell Physiol Biochem Pharmacol (2015) 35 (1):83-91. doi: 10.1159/000369677

16. Mukai T, Hiromatsu Y, Fukutani T, Ichimura M, Kaku H, Miyake I, et al. A C/ $\mathrm{T}$ Polymorphism in the 5' Untranslated Region of the CD40 Gene is Associated With Later Onset of Graves' Disease in Japanese. Endocrine J (2005) 52(4):471-7. doi: 10.1507/endocrj.52.471

17. Krishnappa P, Kong HM, Mohamad IB, Voon K, Somanath SD. CD40 Polymorphism in Cervical Carcinoma in a Subset of Malaysian Population. J Obstetrics Gynaecol Res (2017) 43(5):923-8. doi: 10.1111/jog.13277

18. Shuang C, Dalin L, Weiguang Y, Zhenkun F, Fengyan X, Da P, et al. Association of CD40 Gene Polymorphisms With Sporadic Breast Cancer in Chinese Han Women of Northeast China. PloS One (2011) 6(8):e23762. doi: 10.1371/journal.pone.0023762

19. Zhou G, Wang Y, Fang Z, Liu R, Wang A, Zhao F, et al. CD40 -1c > T Polymorphism and the Risk of Lung Cancer in a Chinese Population. Int $J$ Clin Exp Pathol (2015) 8(11):15163-9.

20. Jasek M, Bojarska-Junak A, Wagner M, Sobczynski M, Wolowiec D, Rolinski J, et al. Association of Variants in BAFF (Rs9514828 and Rs1041569) and BAFF-R (Rs61756766) Genes With the Risk of Chronic Lymphocytic Leukemia. Tumour Biol: J Int Soc Oncodevelopmental Biol Med (2016) 37 (10):13617-26. doi: 10.1007/s13277-016-5182-z

21. Jasek M, Wagner M, Sobczynski M, Wolowiec D, Kuliczkowski K, Woszczyk D, et al. Polymorphisms in Genes of the BAFF/APRIL System May Constitute 
Risk Factors of B-CLL-a Preliminary Study on a Polish Population. Tissue Antigens (2015) 86(4):279-84. doi: 10.1111/tan.12641

22. Tian C, Hromatka BS, Kiefer AK, Eriksson N, Noble SM, Tung JY, et al. Genome-Wide Association and HLA Region Fine-Mapping Studies Identify Susceptibility Loci for Multiple Common Infections. Nat Commun (2017) 8 (1):599. doi: 10.1038/s41467-017-00257-5

23. Hinks A, Cobb J, Marion MC, Prahalad S, Sudman M, Bowes J, et al. Dense Genotyping of Immune-Related Disease Regions Identifies 14 New Susceptibility Loci for Juvenile Idiopathic Arthritis. Nat Genet (2013) 45 (6):664-9. doi: 10.1038/ng.2614

24. World Medical A. World Medical Association Declaration of Helsinki: Ethical Principles for Medical Research Involving Human Subjects. JAMA (2013) 310 (20):2191-4. doi: 10.1001/jama.2013.281053

25. Rasmi RR, Sakthivel KM, Guruvayoorappan C. NF-kappaB Inhibitors in Treatment and Prevention of Lung Cancer. Biomed Pharmacother (2020) 130:110569. doi: 10.1016/j.biopha.2020.110569

26. Skibola CF, Nieters A, Bracci PM, Curry JD, Agana L, Skibola DR, et al. A Functional TNFRSF5 Gene Variant Is Associated With Risk of Lymphoma. Blood (2008) 111(8):4348-54. doi: 10.1182/blood-2007-09112144

27. Nagy A, Lanczky A, Menyhart O, Gyorffy B. Validation of miRNA Prognostic Power in Hepatocellular Carcinoma Using Expression Data of Independent Datasets. Sci Rep (2018) 8(1):9227. doi: 10.1038/s41598-018-27521-y
28. Jacobson EM, Concepcion E, Oashi T. Tomer Y. A Graves' Disease-Associated Kozak Sequence Single-Nucleotide Polymorphism Enhances the Efficiency of CD40 Gene Translation: A Case for Translational Pathophysiology. Endocrinol (2005) 146(6):2684-91. doi: 10.1210/en.2004-1617

Conflict of Interest: The authors declare that the research was conducted in the absence of any commercial or financial relationships that could be construed as a potential conflict of interest.

Publisher's Note: All claims expressed in this article are solely those of the authors and do not necessarily represent those of their affiliated organizations, or those of the publisher, the editors and the reviewers. Any product that may be evaluated in this article, or claim that may be made by its manufacturer, is not guaranteed or endorsed by the publisher.

Copyright (๑) 2021 Dimitrakopoulos, Antonacopoulou, Kottorou, Kalofonou, Panagopoulos, Dougenis, Makatsoris, Tzelepi, Koutras and Kalofonos. This is an open-access article distributed under the terms of the Creative Commons Attribution License (CC BY). The use, distribution or reproduction in other forums is permitted, provided the original author(s) and the copyright owner(s) are credited and that the original publication in this journal is cited, in accordance with accepted academic practice. No use, distribution or reproduction is permitted which does not comply with these terms. 